\title{
THE ITHACA INTERPRETATION OF QUANTUM MECHANICS
}

\author{
N. David Mermin \\ Cornell University, Ithaca, New York, U.S.A. \\ Notes for a lecture given at the Golden Jubilee Workshop on Foundations \\ of Quantum Theory, Tata Institute, Bombay, September 9-12, 1996 \\ I list several strong requirements for what I would consider a sensi- \\ ble interpretation of quantum mechanics and I discuss two simple theo- \\ rems. One, as far as I know, is new; the other was only noted a few years \\ ago. Both have important implications for such a sensible interpreta- \\ tion. My talk will not clear everything up; indeed, you may conclude \\ that it has not cleared anything up. But I hope it will provide a different \\ perspective from which to view some old and vexing puzzles (or, if you \\ believe nothing needs to be cleared up, some ancient verities.)
}

\section{Introduction: A Strategy for Constructing an Interpretation.}

I'd like to describe some thoughts about what ought to go into a satisfactory interpretation of quantum mechanics. I do this with considerable trepidation. "Ought to" can be a highly personal business. And I have yet to put all the pieces together in a fully convincing way. Those who feel they understand quantum mechanics may find what I have to say boring and self-indulgent, while those who are bothered by quantum mechanics may find what follows inadequate or even self-contradictory. So you may get nothing out of my talk beyond a description of two elementary theorems. And one, and perhaps even both of the theorems may be already known to you.

I offer this half baked concoction nevertheless because it seems to me the implications of the theorems for the interpretation of quantum mechanics have not been emphasized and deserve some serious exploration. I've been thinking about them on and off for about half a year now, and have found, to my surprise, that they keep resonating in illuminating ways with various aspects of the Copenhagen interpretation that have always struck me as anthropomorphic or obscure. I have been getting sporadic flashes of feeling that I may actually be starting to understand what Bohr was talking about. Sometimes the sensation persists for many minutes. It's a little like a religious experience and what really worries me is that if I am on the right track, then one of these days, perhaps quite soon, the whole business will suddenly become obvious to me, and from then on I will know that Bohr was right but be unable to explain why to anybody else. 
So it's crucial that I try to communicate some of these ideas before they become so clear to me that only I can understand them. The problem, of course, is that my fragmentary vision may be more of a pipe dream than a religious experience - not a satori but a bad trip. I shall take that risk, and I ask for your indulgence.

I have a simple strategy for constructing an interpretation of quantum mechanics: First of all, by "quantum mechanics" I mean quantum mechanics as it is — not some other theory in which the time evolution is modified by non-linear or stochastic terms, nor even the old theory augmented with some new physical entities (like Bohmian particles) which supplement the conventional formalism without altering any of its observable predictions. I have in mind ordinary everyday quantum mechanics.

I myself have never met an interpretation of quantum mechanics I didn't dislike. I shall try to extract something constructive from all these strongly held negative intuitions, by prohibiting from my own interpretation all of the features I have found unreasonable in all the various interpretations I have encountered. These prohibitions are listed as the first five desiderata below.

To live with so many requirements I need room for maneuver. This is provided by adopting, as my sixth and final desideratum, the view that probabilities are objective intrinsic properties of individual physical systems. I freely admit that I cannot give a clear and coherent statement of what this means. The point of my game is to see if I can solve the interpretive puzzles of quantum mechanics, given a primitive, uninterpreted notion of objective probability. If all quantum puzzles can indeed be reduced to the single puzzle of interpreting objective probabilities, I would count that as progress. Indeed since it is only through quantum mechanics that we have acquired any experience of intrinsically probabilistic phenomena, it seems to me highly unlikely that we can make sense of objective probability without first constructing a clear and coherent formulation of quantum mechanics in terms of such probabilities.

\section{Six Desiderata for an Interpretation of Quantum Mechanics.}

Here are my own personal desiderata for a satisfactory interpretation. Most are based on my persistent discomfort with various commonly held claims about the nature of quantum mechanics.

\section{(1) The theory should describe an objective reality independent of observers and their knowledge.}

The maddening thing about the wave-function is the way in which it manages to mix up objective reality and human knowledge. As a clear indication of this murkiness note 
that even today there is coexistence between those who maintain that the wave-function is entirely real and objective - notably advocates of Bohmian mechanics or seekers of a modified quantum mechanics in which wave-function collapse is a ubiquitous real physical phenomenon - and those who maintain, unambiguously with Heisenberg and presumably with Bohr, that the wave-function is nothing more than a concise encapsulation of our knowledge.

A satisfactory interpretation should be unambiguous about what has objective reality and what does not, and what is objectively real should be cleanly separated from what is "known". Indeed, knowledge should not enter at a fundamental level at all.

\section{(2) The concept of measurement should play no fundamental role.}

I agree with John Bell. ${ }^{1}$ There is a world out there, whether or not we choose to poke at it, and it ought to be possible to make unambiguous statements about the character of that world that make no reference to such probes. A satisfactory interpretation of quantum mechanics ought to make it clear why "measurement" keeps getting in the way of straight talk about the natural world; "measurement" ought not to be a part of that straight talk. Measurement should acquire meaning from the theory — not vice-versa.

The view that physics can offer nothing more than an algorithm telling you how to get from a state preparation to the results of a measurement seems to me absurdly anthropocentric; so does limiting what we can observe to what we can produce ("state preparation" being one of the things you can do with a "measurement apparatus"). Physics ought to describe the unobserved unprepared world. "We" shouldn't have to be there at all.

\section{(3) The theory should describe individual systems - not just ensembles.}

The theory should describe individual systems because the world contains individual systems (and is one itself!) and the theory ought to describe the world and its subsystems. Two attitudes lurk behind every ensemble interpretation. The first is a yearning (not always acknowledged) for hidden variables. For the notion that probabilistic theories must be about ensembles implicitly assumes that probability is about ignorance. (The "hidden variables" are whatever it is that we are ignorant of.) But in a non-determinstic world probability has nothing to do with incomplete knowledge, and ought not to require an ensemble of systems for its interpretation.

\footnotetext{
1 Against 'measurement', Physics World, 33-40, August, 1990.
} 
The second motivation for an ensemble interpretation is the intuition that because quantum mechanics is inherently probabilistic, it only needs to make sense as a theory of ensembles. Whether or not probabilities can be given a sensible meaning for individual systems, this motivation is not compelling. For a theory ought to be able to describe as well as predict the behavior of the world. The fact that physics cannot make deterministic predictions about individual systems does not excuse us from pursuing the goal of being able to describe them as they currently are.

\section{(4) The theory should describe small isolated systems without having to invoke interactions with anything external.}

Not only should the theory describe individual systems, but it should be capable of describing small individual systems. We apply quantum mechanics all the time to toy universes having state-spaces of only a few dimensions. I would like not only to be able to do that, as I now can, but to understand what I am talking about when I do it, as I now cannot.

In particular I would like to have a quantum mechanics that does not require the existence of a "classical domain". Nor should it rely on quantum gravity, or radiation escaping to infinity, or interactions with an external environment for its conceptual validity. These complications may be important for the practical matter of explaining why certain probabilities one expects to be tiny are, in fact tiny. But it ought to be possible to deal with high precision and no conceptual murkiness with small parts of the universe if they are to high precision, isolated from the rest.

\section{(5) Objectively real internal properties of an isolated individual system should} not change when something is done to another non-interacting system.

I agree with Einstein: ${ }^{2}$ "On one supposition we should, in my opinion, absolutely hold fast: the real factual situation of the system $\mathcal{S}_{2}$ is independent of what is done with the system $\mathcal{S}_{1}$, which is spatially separated from the former." Indeed, I would take take spatial separation to be just a particularly clear-cut way of establishing the absence of mediating interactions between the two systems, and apply the supposition — generalized Einstein locality - to any two non-interacting systems.

Einstein used his supposition, together with his intuitions about what constituted a real factual situation, to conclude that quantum mechanics offers an incomplete description

2 Albert Einstein: Philosopher-Scientist, ed. P. A. Schillp, Open Court, La Salle, Illinois, 1970, p. 85. 
of physical reality. I propose to explore the converse approach: assume that quantum mechanics does provide a complete description of physical reality, insist on generalized Einstein-locality, and see how this constrains what can be considered physically real.

(6) It suffices (for now) to base the interpretation of quantum mechanics on the (yet to be supplied) interpretation of objective probability.

I am willing at least provisionally to base an interpretation of quantum mechanics on primitive intuitions about the meaning of probability in individual systems.

Quantum mechanics has taught us that probability is more than just a way of dealing systematically with our own ignorance, but a fundamental feature of the physical world. But we do not yet understand objective probability. Popper ${ }^{3}$ insisted that we cannot think correctly about quantum mechanics until we learn how to think correctly about probability as an objective feature of the world - that the interpretation of quantum mechanics had never squarely faced this issue. I think he was right about that, but wrong in maintaining that with his own formulation of objective probability he had cleared up the conceptual puzzles.

I don't have an understanding of objective probability any better than Popper's, but I maintain that if we can make sense of quantum mechanics conditional upon making sense of probability as an objective property of an individual system, then we will have got somewhere. Indeed, I doubt that we can hope to understand objective probability until we have achieved the partial success of making sense of quantum mechanics, modulo such an understanding. Quantum mechanics is our only source of clues about what objective probability might mean, and we will only unearth those clues if we can succeed in making sense of quantum mechanics from such a perspective.

So my attitude is this: Assume that some wise person has come up with an acceptable notion of probabilities as objective properties of individual systems, and see if one can sweep all the puzzles of quantum mechanics — what Popper called the muddle, mysteries, and horrors — under that single accommodating rug.

In summary, these are my Six Desiderata for an interpretation of quantum mechanics:

(1) Is unambiguous about objective reality.

(2) Uses no prior concept of measurement.

(3) Applies to individual systems.

3 Quantum Theory and the Schism in Physics, Rowman and Littlefield, Totowa, New Jersey, 1982. 
(4) Applies to (small) isolated systems.

(5) Satisfies generalized Einstein-locality.

(6) Rests on prior concept of objective probability.

To persuade you that my aspirations are not made entirely of fluff, let me next digress to tell you about two elementary theorems of quantum mechanics that seem only recently to have been noticed.

\section{Two Elementary Theorems}

I shall describe in a naive way two elementary theorems of quantum mechanics, which bear on the interpretive problem. By "naive" I mean that I shall use uncritically terms forbidden by Desideratum (2) like "measurement", "results of a measurement", etc., because they are a code we all understand, and because avoiding them would make the purely mathematical argument much more clumsy. I shall return to more careful talk when I discuss the relevance of these theorems for the interpretation of quantum mechanics.

To motivate the first theorem, consider the simplest possible quantum mechanical system: a single two-state system, represented as the spin of a spin- $\frac{1}{2}$ particle. Let this system be described by the density matrix

$$
W=\frac{1}{2}\left|\uparrow_{z}\right\rangle\left\langle\uparrow_{z}\left|+\frac{1}{2}\right| \downarrow_{z}\right\rangle\left\langle\downarrow_{z}\right| .
$$

This density matrix has many alternative representations. among them being

$$
W=\frac{1}{2}\left|\uparrow_{x}\right\rangle\left\langle\uparrow_{x}\left|+\frac{1}{2}\right| \downarrow_{x}\right\rangle\left\langle\downarrow_{x}\right| .
$$

The first form is usually said to describe a situation in which the system is in the state $\left|\uparrow_{z}\right\rangle$ with probability $\frac{1}{2}$ and in the state $\left|\downarrow_{z}\right\rangle$ with probability $\frac{1}{2}$; the second, a situation in which the equally probable states are $\left|\uparrow_{x}\right\rangle$ and $\left|\downarrow_{x}\right\rangle$.

Is there an objective difference between these two situations? The statistics of all possible measurements one can make are, of course, the same in both cases because the density matrix is the same, but is there nevertheless an objective difference between a spin with a definite but random polarization along $z$ and a definite but random polarization along $x$ ?

There is no agreement on this elementary conceptual point. People who take the quantum state to be an objective property of an individual system would say there is a difference: in one case this objective property is unknown, but is equally likely to be $\left|\uparrow_{z}\right\rangle$ or $\left|\downarrow_{z}\right\rangle$; in the other case it is either $\left|\uparrow_{x}\right\rangle$ or $\left|\downarrow_{x}\right\rangle$. 
But if you accept Desideratum (5) there can be no objective difference. For one can introduce a second two-state system that does not currently interact with the first, taking the two systems to be in the singlet state

$$
|\Psi\rangle=\frac{1}{\sqrt{2}}\left|\uparrow_{z}\right\rangle\left|\downarrow_{z}\right\rangle-\frac{1}{\sqrt{2}}\left|\downarrow_{z}\right\rangle\left|\uparrow_{z}\right\rangle
$$

which can equally well be written

$$
|\Psi\rangle=\frac{1}{\sqrt{2}}\left|\uparrow_{x}\right\rangle\left|\downarrow_{x}\right\rangle-\frac{1}{\sqrt{2}}\left|\downarrow_{x}\right\rangle\left|\uparrow_{x}\right\rangle .
$$

The representation (3) of $|\Psi\rangle$ establishes that one can produce the situation suggested by the representation (1) of $W$ by measuring $\sigma_{z}$ on the non-interacting ancillary system, while the representation (4) establishes that one can produce the situation suggested by (2) by measuring $\sigma_{x}$ on the ancilla. If objectively real internal properties of an isolated individual system are not to depend on what is done to another non-interacting system, then there can be no difference between these two realizations of the density matrix $W$.

This is the position of those who maintain that Einstein-Podolsky-Rosen correlations and Bell's Theorem establish only that there can be no local hidden-variables underlying quantum mechanics, but do not establish that quantum mechanics itself implies nonlocality. I would like to explore where one can get by adhering to this view.

I once thought this peculiar situation - the ability remotely to produce either of two apparently distinct realizations of the same density matrix $W$ - stemmed from the degeneracy of $W$. But this is wrong. Consider, for example, the non-degenerate density matrix

$$
W=p\left|\uparrow_{z}\right\rangle\left\langle\uparrow_{z}|+q| \downarrow_{z}\right\rangle\left\langle\downarrow_{z}\right|
$$

with $p \neq q$, which in spite of its non-degeneracy also has many alternative representations, one of which is

$$
W=\frac{1}{2}|R\rangle\left\langle R\left|+\frac{1}{2}\right| L\right\rangle\langle L|
$$

where and $|R\rangle$ and $|L\rangle$ are the (non-orthogonal) states

$$
|R\rangle=\sqrt{p}\left|\uparrow_{z}\right\rangle+\sqrt{q}\left|\downarrow_{z}\right\rangle
$$

and

$$
|L\rangle=\sqrt{p}\left|\uparrow_{z}\right\rangle-\sqrt{q}\left|\downarrow_{z}\right\rangle \text {. }
$$

To make talking about things simple suppose that the probability $p$ is very much larger than the probability $q=1-p$. Then interpretation (5) of the density matrix describes 
a system that is in the state $\left|\uparrow_{z}\right\rangle$ with high probability and in the state $\left|\downarrow_{z}\right\rangle$ with low probability, while the interpretation (6) describes a system that is with equal probability in one of two non-orthogonal states representing spin along an axis tilted just slightly away from $\mathbf{z}$ in either the direction $\mathbf{x}$ or $-\mathbf{x}$.

Again one can ask whether there is an objective difference between these two apparently quite different situations, and again the answer must be no. For one can now introduce a second non-interacting two-state system with the pair in the state

$$
|\Psi\rangle=\sqrt{p}\left|\uparrow_{z}\right\rangle\left|\uparrow_{z}\right\rangle+\sqrt{q}\left|\downarrow_{z}\right\rangle\left|\downarrow_{z}\right\rangle
$$

which can equally well be written

$$
|\Psi\rangle=\frac{1}{\sqrt{2}}|R\rangle\left|\uparrow_{x}\right\rangle+\frac{1}{\sqrt{2}}|L\rangle\left|\downarrow_{x}\right\rangle
$$

since $\left|\uparrow_{x}\right\rangle$ and $\left|\downarrow_{x}\right\rangle$ are explicitly

$$
\begin{aligned}
& \left|\uparrow_{x}\right\rangle=\frac{1}{\sqrt{2}}\left|\uparrow_{z}\right\rangle+\frac{1}{\sqrt{2}}\left|\downarrow_{z}\right\rangle, \\
& \left|\downarrow_{x}\right\rangle=\frac{1}{\sqrt{2}}\left|\uparrow_{z}\right\rangle-\frac{1}{\sqrt{2}}\left|\downarrow_{z}\right\rangle .
\end{aligned}
$$

One can produce the situation associated with the representation (5) of $W$ by measuring $\sigma_{z}$ on the non-interacting ancilla, while one can produce the situation suggested by (6) by measuring $\sigma_{x}$ on the ancilla.

It is the content of Theorem I that this state of affairs is completely general: ${ }^{4,5,6}$

\section{Theorem I:}

Given an arbitrary system described by a $d$-dimensional density matrix $W$, and given $N$ different interpretations of that density matrix in terms of ensembles of systems in different (not-necessarily orthogonal) pure states, associated with the expansions

$$
W=\sum_{\mu=1}^{D_{n}} p_{\mu}^{(n)}\left|\phi_{\mu}^{(n)}\right\rangle\left\langle\phi_{\mu}^{(n)}\right|, \quad n=1,2, \ldots N
$$

then if $D$ is the largest of the $D_{n}$ there is a state $|\Psi\rangle$ in $d \times D$ dimensions and $N$ different observables $A_{n}$ in the $D$ dimensional ancillary subspace such that measuring the observable

\footnotetext{
4 N. Gisin, Helv. Phys. Acta 62, 363 (1989)

5 L. P. Hughston, R. Jozsa, and W. K. Wootters, Phys. Lett. A 183, 14 (1993).

6 See Appendix A for a proof that is more complete than Gisin's, and conceptually more straightforward than that of Hughston et al.
} 
$A_{n}$ on the ancilla leaves the original $d$-dimensional subsystem in the state $\left|\phi_{\mu}^{(n)}\right\rangle$ with probably $p_{\mu}^{(n)}$.

If you take Desideratum (5) seriously, then there can be no more objective reality to the different possible realizations of a density matrix, then there is to the different possible ways of expanding a pure state in terms of different complete orthonormal sets. This is not to say that the "ignorance interpretation" of a density matrix does not provide a useful technical way to deal with ensembles of systems. But in the case of an individual system the density matrix must be a fundamental and irreducible objective property, whether or not it is a pure state.

The case of EPR correlations has made familiar the fact that when a system is in a pure state that is not a simple product over subsystems, then its subsystems can have no pure states of their own. As far as I can tell, however, there is no consensus on whether to take the subsystem density matrices as complete objective characterizations of their internal properties. In view of Theorem I, Desideratum (5) requires us to do so. ${ }^{7}$

The second theorem also applies to EPR correlations, but will be used here in a much more general context. To motivate it consider two spin- $\frac{1}{2}$ particles in the singlet state $|\phi\rangle$. Famously, their spin components are perfectly anti-correlated. In particular

$$
\left\langle\phi\left|\sigma_{\mu}^{(1)} \sigma_{\mu}^{(2)}\right| \phi\right\rangle=-1, \quad \mu=x, y, z .
$$

There is a (less famous) coverse of (13):

If a system consisting of two spin- $\frac{1}{2}$ particles has a density matrix $W$, and if

$$
\operatorname{tr} W \sigma_{\mu}^{(1)} \sigma_{\mu}^{(2)}=-1, \quad \mu=x, y, z,
$$

then $W$ is necessarily the projection operator on the singlet state:

$$
W=W_{0}=|\phi\rangle\langle\phi|=\frac{1-\sigma^{(1)} \cdot \sigma^{(2)}}{4} .
$$

This is a direct consequence of the fact that $W=W_{0}$ if and only if $\langle\phi|W| \phi\rangle=1$, but if $W$ satisfies (14) then

$$
\langle\phi|W| \phi\rangle=\operatorname{tr} W W_{0}=\operatorname{tr} W\left(\frac{1-\sigma^{(1)} \cdot \sigma^{(2)}}{4}\right)=\frac{1}{4}\left(1-\sum_{\mu=x, y, z} \operatorname{tr} W \sigma_{\mu}^{(1)} \sigma_{\mu}^{(2)}\right)=1 .
$$

7 Note that this same requirement, in a rather different context, alters the character of the "quantum measurement problem": if a pure state for the system-apparatus supersystem is entirely compatible with density matrices for each subsystem, then the von Neumann "collapse" in a measurement is not from a pure state to a mixture, but from viewing the subsystem density matrices as fundamental and irreducible, to viewing them under the conventional ignorance interpretation. 
There is a way of looking at this trivial result that makes it a little surprising. Suppose you have an ensemble of pairs of $\operatorname{spin} \frac{1}{2}$ particles and you want to know if they all have total spin zero. Total spin being a global property of the pair, one way to determine this would be to measure the total spin of enough pairs to convince yourself that you are always going to get the result 0 . But suppose the pairs are so far apart that this is impractical. There is another way. Two people can do a series of separate measurements of the two $x$ components to convince themselves that they are always anti-correlated, and then do the same for the $y$ and $z$ components. In this way they can establish a global property of an entangled state by a series of local measurements together with the exchange of information about the results of those local measurements.

It is the content of Theorem II that this intriguing state of affairs is entirely general: ${ }^{8}$

\section{Theorem II:}

Given a system $\mathcal{S}=\mathcal{S}_{1} \oplus \mathcal{S}_{2}$ with density matrix $W$, then $W$ is completely determined by the values of $\operatorname{tr} W A \otimes B$ for an appropriate set of observable pairs $A, B$, where $A=A \otimes 1$ is an observable of subsystem $\mathcal{S}_{1}$ and $B=1 \otimes B$ is an observable of subsystem $\mathcal{S}_{2}$. The proof is as follows: ${ }^{9}$

Let the $M_{i}$ be a set of hermitian operators that form a basis for the algebra of operators on the subsystem $\mathcal{S}_{1}$ and let the $N_{i}$ be a similar set for $\mathcal{S}_{2}$. (If the state space for $\mathcal{S}_{1}$ is given an orthonormal basis of states $\left|\psi_{\mu}\right\rangle$ then the $M_{i}$ could, for example consist of all the operators $\left|\psi_{\mu}\right\rangle\left\langle\psi_{\nu}|+| \psi_{\nu}\right\rangle\left\langle\psi_{\mu}\right|$ and all the operators $i\left(\left|\psi_{\mu}\right\rangle\left\langle\psi_{\nu}|-| \psi_{\nu}\right\rangle\left\langle\psi_{\mu}\right|.\right)$ Since the set of all $M_{i} \otimes N_{j}$ is a basis of hermitian operators for the algebra of operators on the full system $\mathcal{S}$, it follows that if $|\phi\rangle$ is any state of $\mathcal{S}$ then the projection operator on $\phi$ has an expansion of the form

$$
|\phi\rangle\langle\phi|=\sum_{i, j} c_{i j}(\phi) M_{i} \otimes N_{j},
$$

where the coefficients $c_{i j}$ are (real) numbers that can be explicitly calculated for any state $|\phi\rangle$ and any choice of the sets of operators $M_{i}$ and $N_{i}$. So if $W$ is the density matrix of $\mathcal{S}$ then

$$
\langle\phi|W| \phi\rangle=\sum_{i, j} c_{i j}(\phi) \operatorname{tr} W M_{i} \otimes N_{j} .
$$

Therefore one can determine any diagonal matrix element of the density matrix $W$ of an ensemble of systems $\mathcal{S}=\mathcal{S}_{1} \oplus \mathcal{S}_{2}$ from the correlations in the results of an appropriate

8 N. D. Mermin, Cornell lecture notes (unpublished), 1995. This must have been noticed before, but I have not yet unearthed it in the literature.

9 I give the argument only for finite dimensional state spaces, leaving the extension to the infinite dimensional case to those more mathematically knowledgeable than I am. 
series of measurements of observables specific to the subsystems $\mathcal{S}_{1}$ and $\mathcal{S}_{2}$. Since an arbitrary off-diagonal matrix element can be expressed in terms of diagonal ones,

$$
\langle\beta|W| \alpha\rangle=\frac{1}{2}\langle\alpha+\beta|W| \alpha+\beta\rangle+\frac{i}{2}\langle\alpha+i \beta|W| \alpha+i \beta\rangle-\frac{1+i}{2}(\langle\alpha|W| \alpha\rangle+\langle\beta|W| \beta\rangle)
$$

we can determine in this way all the matrix elements of the density matrix $W$ in some complete orthonormal basis for $\mathcal{S}$, and hence determine $W$ itself.

This proof easily generalizes to a system $\mathcal{S}=\mathcal{S}_{1} \oplus \cdots \oplus \mathcal{S}_{n}$ composed of more than two subsystems: given any resolution of $\mathcal{S}$ into $n$ subsystems, the density matrix of $\mathcal{S}$ is entirely determined by the correlations among appropriate observables belonging to those subsystems. In such cases the structure of quantum mechanics guarantees the important fact that it doesn't matter whether we pin down the density matrix, for example, of $\mathcal{S}=\mathcal{S}_{1} \oplus \mathcal{S}_{2} \oplus \mathcal{S}_{3}$ from correlations between observables of $\mathcal{S}_{1}$ with observables that act globally on $\mathcal{S}_{2} \oplus \mathcal{S}_{3}$, or from correlations between observables of $\mathcal{S}_{3}$ with observables acting globally on $\mathcal{S}_{1} \oplus \mathcal{S}_{2}$, or from tripartite correlations between observables acting only on the three subsystems.

Thus the density matrix of a composite system determines all the correlations among the subsystems that make it up and, conversely, the correlations among all the subsystems completely determine the density matrix for the composite system they make up. The mathematical structure of quantum mechanics imposes constraints, of course, on what those correlations can be - namely they are restricted to those that can arise from some global density matrix. ${ }^{10}$ The particular form of that density matrix is then completely pinned down by the correlations themselves.

This is familiar in the case $n=1$, where it reduces to the fact that the set of all mean values over the entire system determines the density matrix. What seems to have been overlooked, and what Theorem II establishes is the additional fact that for any resolution of $\mathcal{S}$ into non-trivial subsystems $\mathcal{S}_{1}, \ldots, \mathcal{S}_{n}$, it suffices to determine $W$ to know those mean values only for a set of observables restricted to those of the form $A_{1} \otimes \cdots \otimes A_{n}$ where $A_{j}$ acts only on $\mathcal{S}_{j}$.

In the context of the Six Desiderata, Theorem I asserts that the fundamental irreducible objective character of an individual system is entirely specified by its density

10 That they cannot be more general than that is the content of Gleason's Theorem. It would be interesting to explore the extent to which the underlying structure of probabilities assigned to subspaces of a Hilbert space on which Gleason's Theorem rests is itself pinned down by the requirement of consistency among different possible resolutions of a system into subsystems. 
matrix, and Theorem II then tells us that the fundamental irreducible objective character of an individual system is entirely specified by all the correlations among any particular set of the subsystems into which it can be decomposed.

\section{The Ithaca Interpretation of Quantum Mechanics}

Having only begun looking at quantum mechanics from the point of view of my six Desiderata and two Theorems, I have only scattered, incomplete conclusions to report. At this stage the Ithaca Interpretation is rather fragmentary. Central to it is the doctrine that the only proper subjects of physics are correlations among different parts of the physical world. Correlations are fundamental, irreducible, and objective. They constitute the full content of physical reality. There is no absolute state of being; there are only correlations between subsystems.

Once it occurs to you to put it this way it sounds like a trivial point. For how could it be otherwise? One might imagine a God existing outside of the World with direct unfathomable Access to its Genuine Essence. But physics is more modest in its scope than theology. It aims to understand the world in the world's own terms, and therefore aims only to relate some parts of the world to others. For physicists, if not for theologians, this reduction in scope ought not to be a serious limitation.

If correlations are the fundamental, irreducible, objective components of physical reality, and physical reality consists of individual systems, then probabilities are fundamental, irreducible, objective properties of individual systems. For among the possible correlations among subsystems are those between projection operators associated with the subsystems, which have an immediate interpretation as joint probability distributions. This raises difficult questions about the meaning of probability for individual systems. As I noted at the outset, the strategy of the Ithaca interpretation is to set aside such questions, not because they are unimportant, but because the interpretation of quantum mechanics has enough problems of its own. My aim is to find a satisfactory interpretation of quantum mechanics contingent upon finding a satisfactory understanding of objective probability as a property of individual systems. I would consider that progress.

The question that cannot be evaded, however, is correlations between what? I claim that the failure explicitly to formulate and address this question or to give it only partial answers, is responsible for many of the most notorious difficulties and anthropomorphisms of the Copenhagen interpretation: the claim that the existence of a classical domain is essential for a proper formulation of quantum mechanics; the intrusion at a fundamental level of notions like observation, measurement, or state preparation, into what ought to be a description of phenomena in the unobserved, unmeasured, unprepared natural world; 
and the murkiness of the distinction between objective fact and human knowledge.

To see how this comes about, note that if correlations between subsystems of a closed system are indeed the only proper subjects for physics then the simplest closed non-trivial quantum mechanical system is not a two-state system, but a four-state system, for a two-state (or three-state) system cannot describe two non-trivial subsystems. What is real and objective about such a four-state universe are only the correlations that exist between the pair of two-state subsystems it contains. Observables of one subsystem have no inherent meaning. They acquire such meaning as they have only from the character of their correlations with observables of the other subsystem. If the entire universe consistented of a two-site spin- $\frac{1}{2}$ Heisenberg model the complete objective facts about that universe would be subsumed by the density matrix of that Heisenberg model - i.e. by nothing more or less than the collection of all the correlations between the two subsystems. To ask about the nature of the correlated quantities is to go outside of the universe, for it can only be to ask how they are correlated with something else, and in this toy universe there $i s$ nothing else.

And that's all there is to it for a pair of two-state systems. ${ }^{11}$ Other toy universes are, of course, more complicated, but what is real and objective about them is nothing more or less than all the correlations among their subsystems. What's real about the Universe (if you insist on talking about the Universe) are the correlations among its subsystems.

These correlations constitute the totality of the internal objective reality of individual systems. So what do measurement, or a classical domain, or knowledge have to do with objective reality? Nothing - nothing whatever. They have to do with us.

We're big complicated systems, and we've evolved under the pressure of having to deal with other big complicated systems. We understand them, we can apprehend them, and we've developed language, to represent them to ourselves or to help us tell each other about them. But we did not evolve having to deal with simple two level systems or even complicated atoms. So the only way we can cope with such systems, which evolution did not outfit us to apprehend directly, is to arrange for them to be subsets of larger systems containing subsystems of the kind we do know something about dealing with. We can then learn about the objectively real correlations that exist between the small and the big subsystems, and try to infer the nature of the systems inaccessible to our intuition from how they correlate with the systems we're equipped to deal with. The larger systems are called "classical", and the process of arranging to correlate them with the smaller systems

11 See Appendix B for some of the requirements even so simple a system imposes on the character of objective probabilities. 
is called "the measurement process".

In the measurement process as I've just described it, we ourselves play the role of God, outside of the universe and directly perceiving these informative correlations. It's really not like that, of course. To put the point more accurately it's necessary to acknowledge that we ourselves are physical systems, and what actually emerges from a measurement are the tripartite correlations between us, the classical subsystem, and the inaccessible subsystem. It is because we have developed the ability to make sense of some of the correlations between ourselves and classical systems, that we get something useful out of this process. But this is a property of us - not of the inanimate physical world. Measurement, the classical world, and human knowledge enter the picture only when we ask how we can extract information about the correlations that constitute the world. The correlations themselves, however, are there whether or not we take the trouble to learn about them.

The question of how we are able to understand correlations between ourselves and the accessible "classical" systems we have arranged to correlate with the inaccessible "quantum" systems is known as the problem of consciousness. It's a very difficult problem much more difficult, in my opinion, than the interpretation of quantum mechanics. But it is a problem about us. It is not a problem that has anything to do with what is objectively real about those parts of the physical world that can be well isolated from us.

If the first pillar of the Ithaca Interpretation is that correlations are the only fundamental and objective properties of the world, the second is that the density matrix of a system is a fundamental objective property of that system whether or not it is a onedimensional projection operator. To put it another way, in a nomenclature almost designed to obscure the point, "mixed" states are as fundamental as "pure" states. This flies in the face of much textbook talk about density matrices.

The problem, of course, is that density matrices can serve two purposes. One may indeed be dealing with an ensemble of isolated systems, each of which has a one-dimensional projection operator as its density matrix, and want to average over the ensemble the internal correlations that prevail in each of the subsystems. The mathematical object you need to do this has exactly the same structure, but not at all the same significance, as the fundamental irreducible density matrix of an individual system. It is the latter density matrix that fully describes all the internal correlations of one of the members of a single EPR pair.

It remains to be seen whether this point of view toward density matrices can be developed without running into trouble. It will be important that the development of the Ithaca interpretation must be in a framework that makes it possible to formulate everything 
entirely in terms of internal correlations of isolated individual systems. My guess is that this will be enough to make everything work. Certain common but obscure statements about pure vs. mixed states already make straightforward sense in this new framework. For example it is often said that the difference between a pure state and a mixed state is that in the former case "we" have maximal "knowledge" about the system, while in the latter case "we" do not "know" everything that can be "known". The anthropomorphisms disappear completely if one states this in terms of correlations between subsystems:

The density matrix of a subsystem $\mathcal{S}_{1}$ can be a one-dimensional projection operator (i.e. a pure state) if and only if the only larger systems $\mathcal{S}=\mathcal{S}_{1} \oplus \mathcal{S}_{2}$ that can contain $\mathcal{S}_{1}$ as a subsystem admit of no correlations whatever between $\mathcal{S}_{1}$ and $\mathcal{S}_{2}$. The absence of such correlations is the objective fact. The anthropomorphisms simply express the consequences of this fact for us, should we wish to learn about $\mathcal{S}_{1}$.

It is the program of the Ithaca interpretation to reduce all "quantum mysteries and horrors" to such statements about objective probabilities of individual systems.

By not making it explicit that the pure state of a system (when it has one - and the density matrix, when it does not) is nothing more than a concise way to summarize and reveal the consistency of all the correlations among its subsystems, the Copenhagen interpretation leaves a conceptual vacuum that is often filled with the implicit and sometimes explicit notion that its pure quantum state is a fundamental and irreducible property of a system under study, or even of the entire world. By conferring physical reality on the quantum state one creates a major part of the quantum measurement problem. I am not claiming at this point that granting reality only to correlations among subsystems solves the measurement problem, but it certainly makes it harder to state just what the problem is. Because everything you can formulate in terms of state vectors can also be stated entirely in terms of correlations between subsystems - i.e. in terms of probability distributions - if a quantum measurement problem remains it is going to be a problem about the nature of objective probabilities of individual systems.

It is my optimistic expectation that by making the effort to reformulate the "measurement problem" in those terms one will either demonstrate that it has vanished, or learn something new and important about the nature of objective probability.

\section{Acknowledgments}

I first encountered the view that correlations are fundamental and irreducible when I heard it advocated as the proper way to think about Einstein-Podolsky-Rosen correla- 
tions, in talks by Paul Teller and Arthur Fine. ${ }^{12}$ It did not then occur to me that this might be the proper way to think about much more general correlations. ${ }^{13}$ Nor did it occur to me that objective reality might consist only of correlations until I heard Lee Smolin ${ }^{14}$ sketch an approach to quantum mechanics that treated symmetrically a physical system and the world external to that physical system. Shortly thereafter I received a paper from Carlo Rovelli, ${ }^{15}$ arguing from a very different point of view that quantum states were nothing more than expressions of relations between subsystems. A similar point of view toward quantum states goes at least back to Everett's original "relative-state" formulation of quantum mechanics ${ }^{16}$ before it was swept off into the many-worlds extravaganza. ${ }^{17} \mathrm{I}$ acquired the notion that certain density matrices were just as fundamental and irreducible as pure states from Rudolf Peierls, who insisted to me several years ago that the proper conclusion to draw from EPR was not non-locality, but the absence of any objective difference between mixtures of photons with random 0-90 degree polarizations, or random 45-135 degree polarizations. After the Bielefeld conference I had an instructive e-mail argument with Tim Maudlin about this point, and about some analysis by Sandu Popescu ${ }^{18}$ that confirmed my growing suspicion that conventional views about density matrices and "quantum non-locality" were inadequate. I have also learned from David Albert, John Bell, Rob Clifton, Jörg Dräger, Anupam Garg, Gian-Carlo Ghirardi, Nicholas Gisin, Kurt Gottfried, Dan Greenberger, Bob Griffiths, Lucien Hardy, Jon Jarrett, Thomas Jordan, Tony Leggett, Yuri Orlov, Phillip Pearle, Asher Peres, Oreste Piccioni, Abner Shimony, and Henry Stapp. This work has been supported by the National Science Foundation, Grant No. PHY-9320831.

12 Published in Philosophical Consequences of Quantum Theory, James T. Cushing and Ernan McMullin, eds., Notre Dame Press, Notre Dame, Indiana, 1989.

13 But it should have. This seems to be the point of Bohr's reply to EPR: namely, that there is nothing new or unusual about EPR correlations; precisely the same kinds of correlations are set up in the measurement process, and therefore there is no cause for alarm because he, Bohr, has already straightened that out.

14 Lecture in Bielefeld Germany, August 1995 (unpublished).

15 To appear in International Journal of Modern Physics, 35, No. 8 (1996). See also xxx.lanl.gov e-Print archive, quant-ph/9609002.

16 Hugh Everett, III, Revs. Mod. Phys. 29, 454-462 (1957). See also the gloss on Everett in the paper by John Wheeler that follows, 151-153.

17 Many worlds might threaten practitioners of the Ithaca interpretation when they've cleared up the problems of quantum mechanics and start worrying about how to understand objective probability, but I trust they will by then be wise enough to avoid them.

18 Physical Review Letters 74, 2619-2622 (1995). 


\section{Appendix A: Remote Construction of Arbitrary Ensembles With a Given Density Matrix}

Any density matrix $W$ is hermitian and can therefore be expressed in terms of the orthonormal (but not necessarily complete) set $\left|\phi_{i}\right\rangle$ of its eigenvectors with non-zero eigenvalues:

$$
W=\sum_{i=1}^{d} p_{i}\left|\phi_{i}\right\rangle\left\langle\phi_{i}\right|
$$

(with all $p_{i}>0$.) There are alternative ways to interpret $W$ as distributions of pure states, each of the form:

$$
W=\sum_{\mu=1}^{D} q_{\mu}\left|\psi_{\mu}\right\rangle\left\langle\psi_{\mu}\right|,
$$

where $D \geq d$, and the (normalized) states $\left|\psi_{\mu}\right\rangle$ are not in general orthogonal.

The $\left|\psi_{\mu}\right\rangle$ must span the same space as the $\left|\phi_{i}\right\rangle$, since the spaces spanned by either set have an orthogonal complement which is just the set of all $|\chi\rangle$ with $\langle\chi|W| \chi\rangle=0$.

Consequently there is an expansion

$$
\sqrt{q_{\mu}}\left|\psi_{\mu}\right\rangle=\sum_{i=1}^{d} M_{\mu i} \sqrt{p_{i}}\left|\phi_{i}\right\rangle .
$$

Because the $\left|\phi_{i}\right\rangle$ are an orthonormal set, for (20) and (21) to yield the same density matrix $W$ we must have

$$
\sum_{\mu=1}^{D} M_{\mu i} M_{\mu j}^{*}=\delta_{i j}
$$

If $D>d$ we can extend $M$ to a $D$-dimensional unitary matrix ${ }^{19} U$ with

$$
U_{\mu \nu}=M_{\mu \nu}, \quad \nu \leq d .
$$

It follows from (22) and the unitarity of $U$ that

$$
\sum_{\mu=1}^{D} U_{\mu \nu}^{*} \sqrt{q_{\mu}}\left|\psi_{\mu}\right\rangle=0, \quad \nu>d .
$$

We now define a state in the product of our original state space and a space of dimension $D$ :

$$
|\Phi\rangle=\sum_{i=1}^{d} \sqrt{p_{i}}\left|\phi_{i}\right\rangle \otimes\left|\alpha_{i}\right\rangle,
$$

19 This is simply the assertion that $d$ orthonormal complex $D$-vectors $M_{\mu 1}, \ldots, M_{\mu d}$ can be extended to an orthonormal basis $U_{\mu 1}, \ldots, U_{\mu D}$ for the entire $D$-dimensional space. 
where the $\left|\alpha_{i}\right\rangle$ are the first $d$ members of an (arbitrarily chosen) orthonormal set $\left|\alpha_{\mu}\right\rangle$, $\mu=1 \ldots D$.

It follows from (22) and (23) that

$$
\sqrt{p_{i}}\left|\phi_{i}\right\rangle=\sum_{\mu=1}^{D} \sqrt{q_{\mu}}\left|\psi_{\mu}\right\rangle M_{\mu i}^{*},
$$

and therefore

$$
|\Phi\rangle=\sum_{\mu=1}^{D} \sqrt{q_{\mu}}\left|\psi_{\mu}\right\rangle \sum_{i=1}^{d} M_{\mu i}^{*} \otimes\left|\alpha_{i}\right\rangle .
$$

Eq. (25) permits us to extend the sum to the entire set of $D$ vectors $\left|\alpha_{\mu}\right\rangle$ :

$$
|\Phi\rangle=\sum_{\mu=1}^{D} \sqrt{q_{\mu}}\left|\psi_{\mu}\right\rangle \sum_{\nu=1}^{D} U_{\mu \nu}^{*} \otimes\left|\alpha_{\nu}\right\rangle .
$$

We have thus arrived at an alternative form

$$
|\Phi\rangle=\sum_{\mu=1}^{D} \sqrt{q_{\mu}}\left|\psi_{\mu}\right\rangle \otimes\left|\beta_{\mu}\right\rangle,
$$

where

$$
\left|\beta_{\mu}\right\rangle=\sum_{\nu=1}^{D} U_{\mu \nu}^{*}\left|\alpha_{\nu}\right\rangle .
$$

It follows from the unitarity of $U$ and the orthonormality of the $\left|\alpha_{\mu}\right\rangle$ that the $\left|\beta_{\mu}\right\rangle$ are also an orthonormal set.

If we are given a large number of alternative realizations of $W$ of the form (21), we can take the dimension of the auxilliary space to be the largest $D$ associated with them. The above argument then shows that if we are given any state $|\Phi\rangle$ of the form (26), we can find a representation of $|\Phi\rangle$ having the form (30) for any of the many sets of $\left|\psi_{\mu}\right\rangle$ satisfying (21). By measuring in the auxilliary space an observable whose eigenstates are the associated $\left|\beta_{\mu}\right\rangle$, we can therefore produce an ensemble in the original space in which the system is in the state $\left|\psi_{\mu}\right\rangle$ with probability $q_{\mu}$.

\section{Appendix B: The Hardy Paradox.}

The simplest possible non-trivial closed individual quantum system - a pair of two two-state systems - already gives some useful clues about some of the properties objective probabilities will have to possess. The following example, invented by Lucien Hardy to give 
a particularly powerful version of Bell's Theorem, also enables one to make an important point about objective probabilities.

Call the two two-state subsystems $A$ and $B$. To make the point we need consider only two observables of each system, called $1_{A}, 2_{A}, 1_{B}$, and $2_{B}$. We can label the two eigenstates of each of these observers by a color: red $(R)$ or green $(G)$. In each subsystem take the eigenstates of observable 1 to be non-trivially different from those of observable 2 - i.e. $|1 R\rangle$ is a superposition of $|2 R\rangle$ and $|2 G\rangle$ with both coefficients non-zero. To make the point it suffices to take the symmetric case in which the values of the two coefficients are the same, whether the observables 1 and 2 are associated with subsystem $A$ or subsystem $B$. To keep the notation from getting too cumbersome we abbreviate the designation of a state of the form $\left|1_{A} R\right\rangle \otimes\left|2_{B} G\right\rangle$ (for example) simply to $|1 R, 2 G\rangle$.

Now consider the universe consisting of the pair of two-state systems characterized by the density matrix $|\Psi\rangle\langle\Psi|$ which projects on the (normalized) state:

$$
|\Psi\rangle=\frac{|2 R, 2 R\rangle-|1 R, 1 R\rangle\langle 1 R, 1 R \mid 2 R, 2 R\rangle}{\sqrt{1-\langle 1 R \mid 2 R\rangle^{4}}} .
$$

Clearly

$$
\begin{aligned}
& p(1 R, 1 R)=|\langle 1 R, 1 R \mid \Psi\rangle|^{2}=0, \\
& p(2 G, 1 G)=|\langle 2 G, 1 G \mid \Psi\rangle|^{2}=0, \\
& p(1 G, 2 G)=|\langle 1 G, 2 G \mid \Psi\rangle|^{2}=0,
\end{aligned}
$$

while

$$
p(2 G, 2 G)=|\langle 2 G, 2 G \mid \Psi\rangle|^{2}=\frac{(1-x)^{2} x^{2}}{1-x^{2}}=x^{2}\left(\frac{1-x}{1+x}\right),
$$

where

$$
x=|\langle 1 R \mid 2 R\rangle|^{2} \neq 0 .
$$

The only important thing to note is that the first three of these probabilities are zero and the fourth is non-zero, but I cannot resist noting that the probability $p(2 G, 2 G)$ happens to be maximum when $x=1 / \tau$ (where $\tau$ is the golden mean, $\tau=\frac{\sqrt{5}+1}{2}$ ), in which case the values of all the probabilities associated with the four pairs of subsystem observables are as in the following lovely Table: 


\begin{tabular}{|c|c|c|c|c|}
\hline$p$ & 22 & 11 & 12 & 21 \\
\hline $\mathrm{GG}$ & $\tau^{-5}$ & $\tau^{-3}$ & 0 & 0 \\
\hline $\mathrm{GR}$ & $\tau^{-4}$ & $\tau^{-2}$ & $\tau^{-1}$ & $\tau^{-3}$ \\
\hline $\mathrm{RG}$ & $\tau^{-4}$ & $\tau^{-2}$ & $\tau^{-3}$ & $\tau^{-1}$ \\
\hline $\mathrm{RR}$ & $\tau^{-1}$ & 0 & $\tau^{-4}$ & $\tau^{-4}$ \\
\hline
\end{tabular}

The Hardy paradox consists of observing that the three 0 probabilities translate into three conditional probabilities of unity:

$$
\begin{aligned}
& p\left(1_{A} G, 2_{B} G\right)=0 \Longrightarrow p\left(2_{B} G\right)=p\left(1_{A} R, 2_{B} G\right) \Longrightarrow p\left(1_{A} R \mid 2_{B} G\right)=1 \\
& p\left(1_{A} R, 1_{B} R\right)=0 \Longrightarrow p\left(1_{A} R\right)=p\left(1_{A} R, 1_{B} G\right) \Longrightarrow p\left(1_{B} G \mid 1_{A} R\right)=1 \\
& p\left(2_{A} G, 1_{B} G\right)=0 \Longrightarrow p\left(1_{B} G\right)=p\left(1_{B} G, 2_{A} R\right) \Longrightarrow p\left(2_{A} R \mid 1_{B} G\right)=1 .
\end{aligned}
$$

From these unit conditional probabilities we conclude that $2_{B} G$ requires $1_{A} R$, that $1_{A} R$ requires $1_{B} G$, and that $1_{B} G$ requires $2_{A} R$. Therefore $2_{B} G$ requires $2_{A} R$ :

$$
p\left(2_{A} R \mid 2_{B} G\right)=1
$$

But this contradicts the fact (36) that

$$
p\left(2_{A} G, 2_{B} G\right) \neq 0
$$

The conventional analysis of what's wrong with this reasoning associates the probabilities with the results of measurements. Thus the probability

$$
p\left(1_{A} R \mid 2_{B} G\right)=1
$$

appearing in (38) must actually be conditioned not only on getting $G$ for a measurement of $2_{B}$, but also on both measurements actually being performed. We should therefore use the expanded form

$$
p\left(1_{A} R \mid 2_{B} G ; 1_{A}, 2_{B}\right)=1 .
$$

The second $2_{B}$ is unnecessary, if we interpret $2_{B} G$ to mean property $2_{B}$ is measured and found to have the value $G$. It might appear that the second $1_{A}$ is also unnecessary, but this is incorrect. For the naive argument to go through, the $1_{A} R$ in (38) must mean exactly the same thing as it means in (39) - namely, property $1_{A}$ is measured and found to have 
the value $R$. But the probability is not 1 that if $2_{B}$ is measured and found to have the value $G$ then $1_{A}$ is measured and found to have the value $R$. To get a probability of 1 we must also condition on subsystem $1_{A}$ actually being measured. Therefore we must rewrite $(38)-(40)$ as

$$
\begin{aligned}
& p\left(1_{A} R \mid 2_{B} G ; 1_{A}\right)=1, \\
& p\left(1_{B} G \mid 1_{A} R ; 1_{B}\right)=1, \\
& p\left(2_{A} R \mid 1_{B} G ; 2_{A}\right)=1,
\end{aligned}
$$

and the chain of reasoning following (40) breaks down.

This way out of Hardy's paradox is not available to the Ithaca interpretation, which insists that quantum mechanics should make sense as a description of the objectively real correlations that exist in a universe consisting entirely of the two two-state systems. In such a universe there are no measurements - only correlations. The additional conditioning on an observable "actually being measured" has no meaning. In the Ithaca interpretation the fallacy in the Hardy paradox can only be that the three "conditional probabilities" equal to unity in (38)-(40) have no meaning. It makes no sense to contemplate the probability that $1_{A}$ is $R$ given that $2_{B}$ is $G$. The unconditional value of an observable for a subsystem cannot be "given" - only correlations between subsystems have objective reality.

It therefore appears that the view of probability underlying the Ithaca interpretation must be anti-Bayesian. At some fundamental level unconditional joint objective probabilities have meaning, but certain conditional probabilities have no meaning, because that upon which they are conditioned has no objective reality. Only correlations - i.e. only joint distributions — have objective reality. 